\title{
Impact of Cyproterone Acetate and Ethinylestradiol on Clomiphene Resistant PCOS Patient with High Serum AMH level
}

\author{
Banu $\mathbf{J}^{1}$, Ishrat $\mathrm{S}^{2}$, Deeba $\mathbf{F}^{3}$, Anowary $\mathbf{S}^{4}$, Alamgir $\mathrm{CF}^{5}$, Darmini $\mathbf{M}^{6}$
}

Conflict of Interest: None

Received: 02.01.2020

Accepted: 05.04 .2020 www.banglajol.info/index.php/JSSMC

\begin{abstract}
:
Background: Polycystic ovary syndrome (PCOS) is the most common endocrinopathy in women of reproductive age. PCOS is characterized by hyperandrogenism and oligomenorrhea, and Polycystic ovary. It is a common cause of female subfertility. Anti-mullerianhormone (AMH) is 2-4 times higher in patients with PCOS compared to normal subfertilety patient.
\end{abstract}

Objective: To assess the impact of metformin and oral contraceptives (OCs) containing cyproterone acetate and ethinyl estradiol on serum anti-Mullerian hormone (AMH) levels, with CC resistant polycystic ovary syndrome (PCOS)

Methods: This prospective randomized controlled trial was conducted at Bangabandhu Sheikh Mujib Medical University(BSMMU), Dhaka during the preoid of January 2019 to june 2019. Already diagnosed patients of polycystic ovary syndrome with subfertility with high serum AMH level more than $5 \mathrm{ng} / \mathrm{ml}$ attending in the OPD of Reproductive Endocrinology and infertility department at BSMMU are the study population. Total 60 subjects fulfilling rotardum criteria and who are treated with 3 cycle CC with 100mg /day for 5 days from day 2 of menstrual cycle but fail to ovulate were recruited for the current study. Those subjects with tubal, and male factors excluded from the study. After enrollment of the patients, randomization will be carried out by blocked randomization method to divide them equally into two groups-Group A number of patients 30 ( treated with $2 \mathrm{mg}$ cyproterone acetate (CPA) and $35 \mathrm{mcg}$ of ethinylestradiol (EE) and Group B number of patients 30 treated with Metformin). Group A patients will receive treatment with $2 \mathrm{mg}$ cyproterone acetate (CPA) and $35 \mathrm{mcg}$ of ethinylestradiol (EE) for 6 months and Group B patients will receive treatment with Metformin for 6 months. After enrollment of the patients, base line hormonal assessment basal level of FSH, LH,thyroid-stimulating hormone (TSH), prolactin (PRL), and testosterone hormones were registered and metabolic assessment included OGTT and fasting insulin level were done, and to identify the changes in clinical and biochemical profile of study population. Clinical evaluation, by history and examination, transvaginal ultrasonography of both group and changes of the above mentioned parameters of both group were recorded after 6 month of completion of treatment.

Results: A Changes in clinical parameters (Acne, BMI, Menestrual cycle) was recorded every three monthly for 6 month. Changes of biochemical parameters (serum LH (D2), serum FSH (D2), serum testosterone, OGTT and fasting insulin), serum AMH were recorded every three monthly for 6 month. Changes of sonographic parameters (ovarian volume, Antral follicular count development, was done monthly for 6 cycles. AMH levels are significantly reduced under treatment with OCP containing $35 \mathrm{mg}$ ethinylestradiol plus $2 \mathrm{mg}$ cyproteroneacetate than metformin. AMH was significantly decreased after treatment with $35 \mathrm{mg}$ ethinylestradiol plus 2 mg cyproterone acetate $(p<0.001$ at 6 months). and treatment with metformin did not significantly affect serum AMH levels. AMH was significantly decreased after treatment with metformin $(p<$ 0.005). Serum LH was and OGTT was significantly decreased in both treatment $p<0.001$.

Conclusion(s:) AMH serum levels were significantly decreased under treatment with $35 \mathrm{mg}$ ethinylestradiol plus $2 \mathrm{mg}$ cyproterone acetate, due to decrease in androgens and suppression of gonadotropins $(L H)$.

1. Prof. Jesmine Banu, Chairman, Department of Reproductive Endocrinology and Infertility, BSMMU, Dhaka.

2. Dr. Shakella Ishrat, Associate Professor, Department of Reproductive Endocrinology and Infertility, BSMMU, Dhaka.

3. Dr. Farzana Deeba, Associate Professor, Department of Reproductive Endocrinology and Infertility, BSMMU, Dhaka.

4. Dr. Shahinara Anowary, Assistant Professor, Department of Reproductive Endocrinology and Infertility, BSMMU, Dhaka.

5. Dr. Chowdhury Faisal Alamgir, Internship Trainee, Mymensingh Medical College, Mymensingh

6. Dr. Maliha Darmini, Internship Trainee, Mymensingh Medical College, Mymensingh

Correspondence to: Prof. Jesmine Banu, Chairman, Department of Reproductive Endocrinology and Infertility, BSMMU, Dhaka-1000. Mobile: +8801713030721, E-mail: drjesminebanu@gmail.com 


\section{Introduction:}

Polycystic ovary syndrome (PCOS), a multifactorial disorder, is the most common female endocrinopathy of reproductive age group as well as a major cause of anovulatory infertility. It is a heterogeneous condition and affects about $5-10 \%$ of female populations [Qiao et al. 2013; Durmont et al. 2015]. Currently, it is thought that PCOS emerges from a complex interaction of genetic and environmental traits [Williams et al. 2016].It is characterized by defective follicular development, maturation and ovulation and also hormonal deregulation including LH,AMH and/or androgen hypersecration. The classic symptoms of the disease are due to hyperandrogenism and chronic anovulation. Both of which have substantial psychological, social and economic consequences: Sirmans et al. 2014; Ruan et al.2017]. PCOS is characterized by hyperandrogenism (hirsutismand/or biochemical hyperandrogenemia) and oligo/anovu-lation, and is also highly associated with obesity and insulin resistance (IR). ${ }^{3}$ Anti-Mu llerian hormone (AMH) is a member of the transforming growth factor-b(TGF-b) superfamily of glycoproteins that has been found to play an important role in chronic anovulation by inhibiting the initial recruitment of primordial follicles and by promoting follicular arrest. Serum AMH level is 2-4 fold higher in women with PCOS than in healthy women; Villarroel et al. 2011]. It is due to increased production of AMH per follicle, not due to increased follicular pool. Cyproterone Acetate plus Ethinylestradiol (CPA/EE) is an oral contraceptive pill (OCP) which contains synthetic estrogen 'Ethinylestradiol' and antiandrogen 'Cyproterone Acetate.have traditionally been the first choice for management of PCOS. [Yildiz et al.2008; Buzney et al.2014].CPA/EE are considered to treat androgen dependent conditions i.e. acne, hirsutism and androgenic alopecia, but not indicated solely for contraceptive purpose [Soriano et al. 2016]. Ruan et al. (2017) reviewed that short-term pretreatment using CPA/EE may increase the fertility and improve pregnancy outcome by reducing serum AMH level.

It has been shown that, serum AMH is a reliable diagnostic tool for PCOS [Jacob et al. 2017] than antral follicle count (AFC) [Casadei et al. 2013] as well as useful to establish treatment protocol and to define the best strategy for ovulation induction in infertile women with PCOS [Agathe et al. 2015.

Women with PCOS are usually treated with an oral contraceptive (CPA/EE), while both lean and obese PCOS with IR might benefit from treatment with metformin. Treatment with CPA/EE s is known to normalise menstrual function and to ameliorate hirsutism and acne, reduces serum AMH level on the opposite, treatment with metformin is beneficial for weight reduction and correction of IR, still the effect on menstrual cycle and hyperandrogenism is rather weak. Few data are available on the impact of the treatment modalities on serum AMH levels in women with PCOS. The aim of the present study was to assess the effect of cyproterone acetate and ethinyl estradiol on serum AMH levels in a well characterized cohort PCOS women in comrasion with of metformin. Metformin administration in patients with PCOS exerts a differential action on the ovarian AMH levels on the basis of ovulatory response. Changes in AMH levels in antral follicular fluid during metformin treatment could be involved in the local mechanisms mediating the ovulatory restoration. Recently Different studies shown that, OCP reduces the AMH level [Kallio et al. 2013]. This could be explained by suppression of LH secretion by OCP.As, luteinizing hormone ( $\mathrm{LH}) \mathrm{can}$ be responsible for the over xpression of AMH and AMH specific type 2 receptors (AMHRII) in lutein GCs in anovulatory PCOS. [Pellat et al. 2007; Catteau-Jonard et al. 2008].So, reduced LH may directly impact on AMH secretion.[Van den Berg et al.2010].There is an increased number of pre-antral and antral follicles in the polycystic ovary, many of which individually produce increased amounts of anti-Müllerian hormone (AMH) compared with those in the normal ovary. It is hypothesized that the high AMH concentrations present in women with PCOS play an integral role in causing anovulation due to its inhibitory influence on the actions of follicle-stimulating hormone, which normally promotes follicular development from the small antral to the ovulatory stage. Further indirect evidence to strengthen this hypothesis can be gathered from the practice of ovulation induction. Those with very high concentrations of circulating AMH are less likely to respond with ovulation to treatment with weight loss (Moran et al., 2007), clomiphene citrate or laparoscopic ovarian drilling (Amer et al., 2009). A possible reason that the metformin is less effective and certainly slower than clomiphene in inducing ovulation is the fact that AMH levels only decrease very slowly during this treatment (Fleming et al., 2005; Fabreques et al., 2011). Finally, AMH concentrations decrease during successful low-dose FSH therapy (Catteau-Jonard et al., 2007). Together, this clinical evidence accentuates the fact that the greater the number of antral and pre antral follicles, the higher the AMH concentrations, the more severe the symptoms of PCOS.

Panidis et al. (2010) showed that CPA/EE significantly reduces serum AMH level by decreasing androgens and suppression of Serum LH compared to metformin. Branigan and Estes (2003) found that 2 months of CPA/ $\mathrm{EE}$ use before repeating $\mathrm{CC}$ treatment in $\mathrm{CC}$ resistant women produced effective ovulation and pregnancy rates.

Salama and Hamza (2019) also found improved ovulation and pregnancy rates by using 42 days pretreatment with $\mathrm{CPA} / \mathrm{EE}$ before ovulation induction with letrozole in CC resistant patient. 


\section{Methods:}

This prospective randomized controlled trial study was conducted at Bangabandhu Sheikh Mujib Medical University(BSMMU), Dhaka during the preoid of January 2019 to june 2019. Already diagnosed patients of polycystic ovary syndrome with subfertility with high serum AMH level more than $5 \mathrm{ng} / \mathrm{ml}$ attending in the OPD of Reproductive Endocrinology and infertility department at BSMMU are the study population. Total 60 subjects fulfilling rotardum criteria and treated with 3 cycle CC with $100 \mathrm{mg} /$ day for 5 days from day 2 of menstrual cycle but fail to ovulate were recruited for the current study. Those subjects with tubal, and male factors excluded from the study. Total 60 PCOS with high serum AMH level patients were included in this study with maintaining inclusion criteria, and among them 6 patients were excluded from the study due to.( tubal factors, and male factor Were considered exclusion criteria for all subjects: an age less than 18 or greater than 35 years; a body mass index (BMI, $\mathrm{kg} / \mathrm{m} 2$ ) less than 18 or greater than 25 ; major medical disorders and/or current or previous use of hormonal and/or metabolic drugs; tubal or male factor infertility or sub-fertility investigated with hysterosalpingography and standard semen analysis. During the study complete history, physical examination, basal level of FSH, LH and testosterone hormones were registered and metabolic assessment included OGTT and fasting insulin level were done, and to identify the changes in clinical, transvaginal ultrasonography and biochemical profile of study population. Clinical evaluation consisted of gynecological examination, Biochemical assessment consisted of complete hormonal, including evaluation of serum follicle stimulating hormone (FSH), luteinizing hormone (LH), thyroid-stimulating hormone (TSH), prolactin (PRL), and metabolic evaluation, including evaluation of fasting glucose and insulin levels. Base line Clinical evaluation consisted of gynecological examination, biochemical parameters and sonographic parameters were recorded .Blood sample were be collected in the early morning during follicular phase (Day 2-3 of spontaneous cycle or progestin induced withdrawal bleeding in case of amenorrhea) for the measurement of serum follicle stimulating were hormone (FSH), luteinizing hormone (LH), total testosterone (T), will be measured by using Chemiluminescent Immunoassay . Serum AMH were be measured by Enzyme Linked Immunosorbant Assay (ELISA).On the day 2-5 of a menstrual cycle, a baseline transvaginal ultrasound was performed with the urinary bladder empty using transvaginal probe. The ovarian volume and antral follicle count (AFC) were assessed. Side by side absence of any ovarian cyst will be ensured before starting the treatment. The volume of each ovary were calculated by measuring the ovarian diameters (D) in three perpendicular directions. Here, the formula of ellipsoid: D1 xD2x D3x 0.5236 will be applied. For AFC, small follicles with a diameter between 2 and $9 \mathrm{~mm}$ will be calculated following the recommendations. After enrollment of the patients, randomization ware carried out by blocked randomization method to divide them equally into two groups- Group A number of patients 30 ( treated with $2 \mathrm{mg}$ cyproterone acetate (CPA) and $35 \mathrm{mcg}$ of ethinylestradiol (EE) and Group B number of patients 30 treated with Metformin). Group A patients will receive treatment with $2 \mathrm{mg}$ cyproterone acetate (CPA) and $35 \mathrm{mcg}$ of ethinylestradiol (EE) for 6 months and Group B patients were receive treatment with Metformin for 6 months. Changes of the above mentioned parameters were recorded of patients after 6 month of treatment. Ciproterone acetate and metformin. The information ware collected and recorded in the present questionnaire.

To asses changes of base line parameter and parameter after 6 month of treatment respectively away 2 group.(Ovarian changes was assessed by transvaginal monitoring antral follicular count and size of follicles Hormonal analysis.)

. The study was prospective and randomised. Randomisation was non-blind and was based on patients' chronological presence at the outpatient endocrine infirmary, namely the first one in Group A the second in Group B etc. sixty (60)women with PCOS were randomly divided into two groups: Group A (n30, age 18-35 years, BMI $19-25 \mathrm{~kg} / \mathrm{m} 2$ ), which comprised women were treated for 6 months with an OC containing 35 mgethinylestradiol plus $2 \mathrm{mg}$ cyproterone acetate.

Group B(30 n, age, BMI same range), which comprised women who were treated for 6months with metformin $500 \mathrm{mg} 8$ hourly.

After treatment clinical evaluation, blood sampling, transvaginal ultrasonography, were done. Blood samples were collected during on the 2-3 day of cycle (reflecting 3 and 6 months of treatment), while patients were on OCP or metformin. Biochemical assessment consisted of complete hormonal, including evaluation of serum follicle stimulating hormone (FSH), luteinizing hormone $(\mathrm{LH})$, thyroid-stimulating hormone (TSH), prolactin (PRL), , total testosterone (T), and metabolic evaluation, including evaluation of fasting glucose and insulin levels. Serum AMH levels were assessed .

Finally, the ovarian dimensions, volume and morphology and the number of antral follicles (follicular diameter ranged from 2 to $9 \mathrm{~mm}$ ) were evaluated bilaterally by 
transvaginal ultrasonography. The antral follicle number per ovary, defined as the average for the total number of antral follicles counted from both ovaries, was also calculated monthly for 6 month.

Changes in clinical parameters (Acne, BMI, Menestraul cycle) was recorded after 6 month. Changes of biochemical parameters (serum LH (D2), serum FSH (D2), serum testosterone, OGTT and fasting insulin), serum AMH were recorded every three monthly for 6 month. Changes of sonographicparameters (ovarian volume, Antral follicular count development, was done after 6 month.

\section{Results:}

This prospective study was carried out in the of Reproductive endocrinology and infertility Department of Bangabandhu Sheikh Mujib Medical University, Dhaka, between January 2019 to june 2019. Total 60 PCOS with high serum AMH level patients were included in this study with maintaining inclusion and exclusion criteria, due to. (tubal factors, and male factor..

During the study complete history, physical examination, basal level of FSH, LH and testosterone hormones were registered and metabolic assessment included OGTT and fasting insulin level were done, and to identify the changes in clinical and biochemical profile and TVS findings following ciproterone acetate in PCOS patients with high AMH level. Statistical analyses were carried out by using the Statistical Package for Social Sciences version 23.0 for Windows (SPSS Inc, Chicago, Illinois, USA) in personal computer. The $\mathrm{p}$ value $<0.05$ were considered as statistically significant

Table 1 shows characteristics of the study population. It was observed that before treatment mean age of study population was found $26.8 \pm 3.6$ years in Group-A and $25.5 \pm 2.8$ in Group-B . Mean BMI was found 26.3 \pm 4.9 $\mathrm{kg} / \mathrm{m} 2$.in Group- A and $25.69 \pm 2.34$ in Group-B. Hirsutism $10.23 \pm 3.56$ in Group- A and $9.59 \pm 1.47$ in Group-B,Ovarian volume $12.34 \pm 1.67$ in Group-A, 11.89 \pm 2.46 in Group-B

Number of follicle14.27 \pm 2.13 in Group-A, $13.45 \pm 1.78$ in Group-B

Table I

Clinical presentations of the study participants.

\begin{tabular}{|c|c|c|}
\hline \multicolumn{3}{|c|}{ Clinical presentations of the study participants. } \\
\hline Parameters & Group-A & Group-B \\
\hline & Mean \pm SD & Mean \pm SD \\
\hline Age (in years) & $26.8 \pm 3.6$ & $25.5 \pm 2.8$ \\
\hline BMI $(\mathrm{Kg} / \mathrm{m} 2)$ & $26.3 \pm 4.9$ & $25.69 \pm 2.34$ \\
\hline Waist circumference $(\mathrm{cm})$ & $88.34 \pm 2.15$ & $87.40 \pm 3.68$ \\
\hline Hirsutism & $10.23 \pm 3.56$ & $9.59 \pm 1.47$ \\
\hline Ovarian volume & $12.34 \pm 1.67$ & $11.89 \pm 2.46$ \\
\hline Number of follicle & $14.27 \pm 2.13$ & $13.45 \pm 1.78$ \\
\hline
\end{tabular}

Table II

Baseline the hormonal parameter of both group (before treatment).

\begin{tabular}{lcc} 
Variables & Group-A & Group-B \\
& Mean \pm SD & Mean \pm SD \\
\hline OGTTmmol/L & $8.7 \pm 1.0$ & $7.13 \pm 2.4$ \\
FSH $(\mathrm{mIU} / \mathrm{ml})$ & $5.40 \pm 1.56$ & $6.04 \pm 1.75$ \\
LH $(\mathrm{mIU} / \mathrm{ml})$ & $14.8 \pm 6.64$ & $13.76 \pm 6.86$ \\
Serum Testosterone $(\mathrm{ng} / \mathrm{ml})$ & $81.79 \pm 33.22$ & $83.14 \pm 22.14$ \\
AMH $(\mathrm{ng} / \mathrm{dl})$ & $9.10 \pm 2.95$ & $8.90 \pm 3.49$ \\
HOMA IR & $1.95 \pm 1 . .4$ & $2.11 \pm 1.18$ \\
\hline
\end{tabular}


Table III

\begin{tabular}{|c|c|c|c|c|}
\hline \multicolumn{5}{|c|}{ Hormonal parameter of both groups after treatment. } \\
\hline Parameter & Group -A & P-Value & Group -B & P-Value \\
\hline Serum LH & $4.02 \pm 1.05$ & $<0.001$ & $7.47 \pm 3.06$ & $=0.001$ \\
\hline Serum FSH & $3.88 \pm 2.35$ & $=0.002$ & $4.14 \pm 6.32$ & $=0.012$ \\
\hline Serum Testesterone & $50.78 \pm 14.86$ & $=0.003$ & $55.59 \pm 27.23$ & $=0.004$ \\
\hline OGTT & $5.07 \pm 2.37$ & $<0.001$ & $6.14 \pm 4.25$ & $<0.001$ \\
\hline HOMA IR & $2.90 \pm 1.76$ & $=0.152$ & $2.60 \pm 1.53$ & $=0.342$ \\
\hline Serum AMH & $3.88 \pm 0.84$ & $<0.001$ & $6.42 \pm 3.57$ & $<0.005$ \\
\hline BMI & $21.05 \pm 1.99$ & 0.967 & $21.67 \pm 2.30$ & 0.990 \\
\hline
\end{tabular}

Table IV

The Mean number of follicles in Group A (CPA/EE) and Group B (Metformin) after treatment.

\begin{tabular}{lcccc} 
Parameter & $\begin{array}{c}\text { Group -A } \\
\text { Mean - SD }\end{array}$ & P-Value & $\begin{array}{c}\text { Group -A } \\
\text { Mean - SD }\end{array}$ & P-Value \\
\hline Total number of follicles & $8.24 \pm 1.46$ & $<.001$ & $10.88 \pm 1.06$ & $<0.05$ \\
Ovarian Volume & $6.12 \pm 1.89$ & $<.001$ & $9.87 \pm 2.02$ & $<0.453$ \\
\hline
\end{tabular}

Total number of follicle and Ovarian Volume was significantly decreased under CA/EE treatment compared to metformin $(\mathrm{p}<0.001)$.

Mean base line OGTT mmol/L was found in $8.7 \pm 1.0$ Group-A ,7.13 \pm 2.4 Group-B .Mean FSH $(\mathrm{mIU} / \mathrm{ml})$ Group-A $5.40 \pm 1.56$ and Group-B $6.04 \pm 1.75 \mathrm{mIU} / \mathrm{ml}$ ) Mean LHmIU/ml level was found in $14.8 \pm 6.64$ GroupA,and13.76 \pm 6.86 Group-B Mean testosterone(ng/ml) was found in $81.79 \pm 33.22$ Group-A, and $83.14 \pm 22.14$ Group-B Mean AMH (ng/dl)9.10, \pm 2.95 Group-A, and $8.90 \pm 3.49$ Group-BHOMA IR $3.95 \pm 1.4$ Group-A and $3.11 \pm 1.18$ Group-B.

\section{Results:}

AMH was significantly decreased after treatment with 35 $\mu$ gethinylestradiol plus $2 \mathrm{mg}$ cyproterone acetate ( $\mathrm{p}$ $<0.001$ at 6 months). and treatment with metformin did not significantly affect serum AMH levels. AMH was significantly decreased under $\mathrm{CA} / \mathrm{EE}$ treatment compared to metformin $(p=0.005)$. Serum LH and OGTT was significantly decreased with both group of treatment $\mathrm{p}<$ 0.001 .

\section{Discussion:}

$\mathrm{AMH}$ is produced by the granulosa cells of early developing follicles ${ }^{17}$ and reflects the continuous, non-cycling growth of small follicles in the ovary. ${ }^{18} \mathrm{AMH}$ has been found to be increased in the serum of women with PCOS. ${ }^{6-10}$. $\mathrm{AMH}$ levels are not influenced by hormone al fluctuations and remain constant throughout the menstrual cycle, making it a promising diagnostic marker for patients with PCOS [19]. The aim of the present study was to assess the impact of CEA/EE on serum AMH levels in women with PCOS. The data of the present study clearly demonstrate asignificant decrease of serum AMH levels under treatment with an OC containing $35 \mathrm{mg}$ ethinylestradiol plus EE while no statistically significant change was noted under treatment with metformin 500 mg 8 hourly. Our data are clearly contra-dictory to the data previously presented by Somunkiranet al. ${ }^{14}$ who reported no change in serum AMH level safter 6 months of treatment with $35 \mathrm{mg}$ ethinylestradiol plus $2 \mathrm{mg}$ cyproterone acetate. This discrepancy might beattributed to the different selection of patients with PCOS between the two studies. Somunkiran et al. recruited their PCOS patients according to the criteria of the Rotterdam PCOS ${ }^{21}$ consensus workshop group, namely the presence of two of the following three criteria: oligomenor-rhea or amenorrhea, clinical or biochemical signs of hyperandrogenism, and ultrasonographic polycystic ovarian morphology ${ }^{14}$, while patients with PCOS in our study were recruited to fulfil (and) the stricter criteri proposed in 1990 by the National Institute of Child Healt hand Human Development Conference on PCOS ${ }^{16}$ Never theless, in the present study, serum AMH levels after 6 months of treatment with $35 \mathrm{mg}$ ethinylestradiol 
plus $2 \mathrm{mg}$ cyproter-one acetate were significantly related to serum testosterone .In a previous study, we have shown that $\mathrm{AMH}$ levels were higher in anovulatory and hyperandrogenemic women with NIH-defined 'classical' PCOS, compared to anovulatory women with PCOS morphology on ultrasound but normal androgen. Serum AMH levels at baseline 6 months treatment with $35 \mathrm{mg}$ ethinylestradiol and $2 \mathrm{mg}$ cyproterone acetate, with metformin $500 \mathrm{mg} 8$ hourly. Mean base line AMH (ng/dl) $9.10 \pm, 2.95$ Group-A, and 8.90 \pm 3.49 Group-B . Serum AMH group A $3.88 \pm 0.84$ p value $<0.001$ after 6 month

Serum AMH Group-B $6.42 \pm 3.57$ and $\mathrm{p}$ value $<0.005$ after 6 month. AMH was significantly decreased after treatment with $35 \mu \mathrm{g}$ ethinylestradiol plus $2 \mathrm{mg}$ cyproterone acetate $(\mathrm{p}<0.001$ at 6 months $)$. and treatment with metformin did not significantly affect serum AMH levels $\mathrm{p}$ value $<0.005$ after 6 month. Levels. In this study, the strong positive association between $\mathrm{LH}$ and AMH levels, the significantly higher LH concentrations in women with 'severe' PCOS along with the highest levels of serum AMH,

Therefore, the findings of the present study add additional evidence that AMH levels reflect the severity of PCOS, traditionally defined by its two cardinal elements, i.e. oligo-anovulation and hyperandro-genemia. ${ }^{16}$ Somunkiran et al. [14 Ovulatory disorders in women with PCOS are caused by an increased early follicular growth, resulting in a large reserve of follicles and/or a defective follicular selection, leading to follicular arrest ${ }^{4}$ Since intra-ovarian andro-gens are also responsible for defective follicular selection and follicular arrest, it has been proposed that the intra-ovarian hyperandrogenism by increasing the AMH intra-ovarian level could exert an inhibiting effect on the selection process [8]. The excess in AMH production by poly cystic ovaries might be the result of the increased number of follicles $2-9 \mathrm{~mm}$ in diameter caused by the intra-ovarian excess of androgens ${ }^{4}$, We have previously shown that increased serum LH levels was the most significant independent link between PCOS-associated disorders of ovulation and the observed increase in serum AMH as it contributed as much as $18 \%$ in the variance of circulating $\mathrm{AMH} .^{7}$ It is postulated that premature LH action on the granulosa cells of anovulatory women with PCOS con-tribute to the follicular arrest, being the link between PCOS-associated disorders of ovulation and the observed increase in ovarian production of AMH. ${ }^{11}$ In the present study BMI was not modified in a both groups after 6 months of treatment, therefore the major effect of metformin was not achieved and thus additional time should be given in or derto observe the net effect of metformin administration on body weight reduction, IR amelioration and eventually its effect on serum AMH levels. Therefore, the findings of this study suggest that although obesity and, to a lesser extent IR, play a role in modulating serum AMH levels, the decrease in.Finally, the results of the present study should beinterpreted with caution taking into account the limitations of this study. A systematic review Badawy et al. 42 and Elmashad43 concluded that ovarian volume has little clinical application in prediction of poor pregnancy response. However, another recent review commented on the value of ovarian volume with regard to its easy execution, and therefore could be included in preparatory protocols providing data for continuity of research.

\section{Conclusion:}

AMH serum levels reflect the severity of PCOS and are significantly increased in its 'classical' phenotypic forms, based on consensus 2015. Moreover, AMH levels are significantly decreased under treatment with OCS containing 35 ethinylestradiol plus $2 \mathrm{mg}$ Cyproteroneacetate, through decrease in serum androgens and the suppression of pituitary gonadotropins (LH )There by releage of negative feedback effect of LH and androgen on hypothalamus is released. So compensatory release of FSH, resulting correction of anovultion which is assessed by folliculometry, So further research is needed to evaluate the response of ovlation induction pretreatment serum AMH level with Ciproterone Acetate and estradiol with Patient PCOS having high seme AMH levels who one clouephane resident.

\section{References}

1. Goodman NF, Cobin RH, Futer weit W, et al. American association of clinical endocrinologists, American college of endocrinology, and androgen excess and PCOS society disease state clinical review: guide to the best practices in the evaluation and treatment of polycystic ovary syndrome-PARTE ndocrinolPract. 2015;21:1415-1426

2. Lizneva D, Suturina L, Walker W, et al. Criteria, prevalence, and phenotypes of polycystic ovary syndrome. Fertil Steril. 2016; 106:6-15.[3]

3. Rotterdam ESHRE/ASRM-sponsored PCOS consensus work shop group. Revised 2003 consensus on diagnostic criteria and long-term health risks related to polycystic ovary syndrome (PCOS).Hum Reprod. 2004; 19:41-47.[4]

4. National Institutes of Health. Evidence-based methodology workshop on polycystic ovary syndrome, December 3-5, 2012.Executive summary [Internet]. Available from: https:// preven-tion.nih.gov/docs/programs/pcos/FinalReport.pdf

5. Sirmans SM, Pate KA. Epidemiology, diagnosis, and management of polycystic ovary syndrome. Clin Epidemiol. 2014;6:1-13.[6] Zafari ZF, Jafarabadi M, Naghizadeh MM, et al.

6. Psychologicaldistress in women with polycystic ovary syndrome from ImamKhomeini Hospital, Tehran. J ReprodInfertil. 2012;13:111-115 
7. Jones GL, Hall JM, Balen AH, et al. Health-related quality of life measurement in women with polycystic ovary syndrome: a systematic review. Hum Reprod Update. 2008;14:15-25

8. Azziz R, Marin C, Hoq L, et al. Health care-related economic bur-den of the polycystic ovary syndrome during the reproductive life span. J Clin Endocrinol Metab. 2005;90:4650-4658.

9. Fauser BCJM, Tarlatzis BC, Rebar RW, et al. Consensus on women's health aspects of polycystic ovary syndrome (PCOS): the Amsterdam ESHRE/ASRM-sponsored 3rd PCOS consensus work-shop group. Fertil Steril. 2012;97:28-38

10. Haoula Z, Salman M, Atiomo W. Evaluating the association between endometrial cancer and polycystic ovary syndrome. Human Reprod. 2012;27:1327-1331.

11. Badawy AS, Elnashar A.Treatment options for polycystic ovarysyndrome. Int J Womens Health. 2011;3:25-35.

12. Poretsky L, Piper B. Insulin resistance, hypersensitivity of LH, and dual defect hypothesis for the pathogenesis of polycysticovary syndrome. Obstet Gynecol. 1994;84:613-621.

13. McCartney CR, Eagleson CR, Marshall JC. Regulation of gonadotropin secretion: implications for polycystic ovary syndrome. Semin Reprod Med. 2002;20:317-336

14. Royal College of Obstetricians and Gynecologists. Long-term consequences of Polycystic Ovary Syndrome. Green-top Guideline No. 33. November 2014 [Internet]. Available from:https://www.rcog.org.uk/en/guidelines-research-services/ guide-lines/gtg33

15. Diamanti-Kandarakis E, Dunaif A. Insulin resistance and the poly cystic ovary syndrome revisited: an update on mechanisms and implications. Endocr Rev. 2012;33:981-1030

16. Zawadski JK, Dunaif A.Diagnostic criteria for poly cysticovary syndrome. In: Dunaif A, Givens JR, Haseltine FP, Merriam GE, editors. Poly cystic ovary syndrome, Mass. USA: Black well Scientific Boston; 1992. pp 377-384.

17. Baarends WM, Uilenbroek JT, Kramer P, HoogerbruggeJW, vanLeeuwen EC, Themmen AP, Grootegoed JA. Anti-Mu llerian hormone and anti-Mu llerian hormone type IIreceptor messenger ribonucleic acid expression in rat ovaries during postnatal development, the estrous cycle, and gonado-tropininduced follicle growth. Endocrinology 1995;136:4951-4962.

18. Visser JA, de Jong FH, Laven JS, Them men AP. Anti-Mu llerian hormone: a new marker for ovarian function. Reproduction 2006;131:1-9.

19. La Marca A, Stabile G, Artenisio AC, Volpe A. Serum anti-Mu llerian hormone throughout the human menstrual cycle. Hum Reprod 2006;21:3103-3107.

20. Nestler JE. Metformin for the treatment of the polycystic ovary syndrome. N Engl J Med 2008;358:47-54.

21. Rotterdam ESHRE/ASRM-sponsored PCOS Consensus Workshop Group Revised 2003 consensus on diagnosticcriteria and long-term health risks related to polycystic ovary syndrome (PCOS). Hum Reprod 2004;19:41-47.

22. Dunaif A, Futter weit W, Segal KR, Dobrjansky A. Profound peripheral insulin resistance, independent of obesity, in the polycystic ovary syndrome. Diabetes 1989;38:1165-1174

23. Dunaif A. Insulin resistance and the polycystic ovary syndrome: mechanism and implication for pathogenesis. EndocrRev 1997; 18:774-800

24. Wu J, Zhu Y, Jiang Y, Cao Y. Effects of metformin and ethinylestradiol-cyproterone acetate on clinical, endocrine and metabolic factors in women with polycystic ovary syndrome. Gynecol Endocrinol 2008;24:392-398.

25. Luque- Ramý ìrez M, Alvarez-Blasco F, Botella-CarreteroJI, Martý ìnez-Bermejo E, Lasuncio ì n MA, Escobar-Morreale HF.Comparison of ethinyl-estradiol plus cyproteroneac etateversus metformin effects on classic metabolic cardiovascularrisk factors in women with the polycystic ovary syndrome. J Clin Endocrinol Metab 2007;92:2453-2456.

26. Carlsen SM, Vanky E, Fleming R. Anti-Mu llerianhormoneconcentrations in androgen-suppressed women with poly cys-tic ovary syndrome. Hum Reprod 2009;24:1732-1738.592D. Panidis et al.Gynecol Endocrinol Downloaded from informahealthcare.com by Hacettepe Univ. on 01/27/12For personal use only. Endocrin Downloaded from informahealthcare.com by Hacettepe Univ. on 01/27/12For personal use only. 